\title{
Computation of $\bar{\kappa}_{3}$
}

In this section, we derive the regular graph approximation for the average joint third cumulant. We start with the following formula.

$$
\begin{aligned}
\bar{\kappa}_{3} & =\frac{\bar{\Lambda}}{N^{3}} \sum_{l_{1}, l_{2}, l_{3}}\left[\sum_{i, j, k, m} G_{i m}^{l_{1}} G_{j m}^{l_{2}} G_{k m}^{l_{3}}\right] \\
& +\frac{3 \bar{\Lambda}}{N^{3}} \sum_{l_{1}, l_{2}}\left[\sum_{i, j, k} G_{i k}^{l_{1}} G_{j k}^{l_{2}}\right] \\
& +\frac{3 \bar{\Lambda}}{N^{3}} \sum_{l_{1}, l_{2}, l_{3}}\left[\sum_{i, j, k, m} G_{i m}^{l_{1}} G_{j m}^{l_{2}} G_{m k}^{l_{3}}\right] \\
& +\frac{6 \bar{\Lambda}}{N^{3}} \sum_{l_{1}, l_{2}, l_{3}}\left[\sum_{i, j, k, n} G_{i j}^{l_{1}} G_{j n}^{l_{2}} G_{k n}^{l_{3}}\right] \\
& +\frac{6 \bar{\Lambda}}{N^{3}} \sum_{l_{1}, l_{2}}\left[\sum_{i, j, k} G_{i j}^{l_{1}} G_{j k}^{l_{2}}\right] \\
& +\frac{3 \bar{\Lambda}}{N^{3}} \sum_{l_{1}, l_{2}, l_{3}, l_{4}}\left[\sum_{i, j, k, m, n} G_{i m}^{l_{1}} G_{j m}^{l_{2}} G_{m n}^{l_{4}} G_{k n}^{l_{3}}\right] .
\end{aligned}
$$

These 6 terms each correspond to one of the 6 possible rooted trees, depicted in Fig. 4. First, let us compute the terms inside the square brackets. The following result holds :

Let $\mathcal{T}$ be an arbitrary rooted tree with at most $N$ nodes. Then, the "square bracket" corresponding to it (obtained by summing over all possible indices of nodes in a tree with a fixed number of branches) can be computed using the following pseudo-algorithm :

1. Set $X=1$.

2. For every leaf of $\mathcal{T}, X \leftarrow X * N$

3. For every edge in $\mathcal{T}, X \leftarrow X * p$

4. For every internal node of out-degree $k$ (where the root counts as an internal node) of $\mathcal{T}, X \leftarrow X * \frac{\mu^{(k)} \cdot N}{p}$, where $\mu^{(k)}$ is defined as the average common input, shared by $k$ nodes, and is defined by

$$
\mu^{(k)}=p \sum_{t} \frac{N_{t}}{N} g_{t}^{k}
$$

It is not difficult to prove that this is equivalent to : 
Let $\mathcal{T}$ be an arbitrary rooted tree with $n \leq N$ nodes and $l \leq n-1$ leaves. Then, the corresponding "square bracket term" is equal to

$$
N^{n} p^{l-1} \prod_{v} \mu^{\left(k_{v}\right)}\left[\mu^{(1)} N\right]^{l_{1}+\cdots+l_{n-1}-n+1},
$$

where the product is over all internal nodes (i.e. nodes that are not leaves) of $\mathcal{T}$ and $k_{v}$ is the out-degree of node $v$. The numbers $l_{1}, \cdots, l_{n-1}$ encode the lengths of branches of $\mathcal{T}$, of which there are $n-1$ (in a tree with $n$ nodes).

We now use the previous result to compute "square bracket terms" for all the trees in Fig. 4. We have

1. $T_{1}:$

$$
X_{1}=N^{4} p^{2} \mu^{(3)}\left[\mu^{(1)} N\right]^{l_{1}+l_{2}+l_{3}-3},
$$

where $l_{1}, l_{2}$ and $l_{3}$ are fixed lengths of the three branches of the first tree and $l_{1}, l_{2}, l_{3} \geq 1$.

2. $T_{2}$ :

$$
X_{2}=N^{3} p \mu^{(2)}\left[\mu^{(1)} N\right]^{l_{1}+l_{2}-2} .
$$

3. $T_{3}, T_{4}$ - they have the same number of nodes and their internal nodes have the same distribution of out-degrees :

$$
X_{3}, X_{4}=N^{4} p \mu^{(1)} \mu^{(2)}\left[\mu^{(1)} N\right]^{l_{1}+l_{2}+l_{3}-3} .
$$

4. $T_{5}:$

$$
X_{5}=N^{3}\left[\mu^{(1)}\right]^{2}\left[\mu^{(1)} N\right]^{l_{1}+l_{2}-2} .
$$

5. $T_{6}:$

$$
X_{6}=N^{5} p^{2}\left[\mu^{(2)}\right]^{2}\left[\mu^{(1)} N\right]^{l_{1}+l_{2}+l_{3}+l_{4}-4} .
$$

Now, we are finally ready to compute the sums of the "bracket terms" over the lengths of branches $\left\{l_{i}\right\}_{1 \leq i \leq k}$. Looking at the expressions for $\left\{X_{k}\right\}_{1 \leq k \leq 6}$, it is easy to see that the only place where the lengths $l_{i}$ explicitly appear is as powers of the term $\mu^{(1)} N$. Thus, to compute sums over lengths of branches, we have to be able to compute

$$
S_{k} \equiv \sum_{l_{1}, l_{2}, \cdots, l_{k}} a^{\sum_{i=1}^{k}\left(l_{i}-1\right)} .
$$

First, we make the following observation.

The sum $S_{k}$ can be written as

$$
S_{k}=\sum_{r \geq k}\left(\begin{array}{l}
r-1 \\
k-1
\end{array}\right) a^{r-k}=\sum_{r \geq k} \frac{1}{(k-1) !}(r-1)(r-2) \cdots(r-k+1) a^{r-k}
$$


To see why this is true, consider that, by definition, $S_{k}=\sum_{l_{1}} \sum_{l_{2}} \cdots \sum_{l_{k}} a^{\sum_{i=1}^{k}\left(l_{1}-1\right)}$. If we introduce a new variable $r=\sum_{i=1}^{k} l_{i}$, we have that $r \geq k$, as $l_{i} \geq 1$, for all $i$. Furthermore, the number of different $k$-tuples $\left(l_{1}, \cdots, l_{k}\right)$ that result in the same value of $r$ is equal to the number of compositions of the number $r$ into $k$ parts, which is equal to $\left(\begin{array}{c}r-1 \\ k-1\end{array}\right)$. The claim then readily follows.

In addition, we also have the following :

The sum $S_{k}$ is explicitly summable for all $|a|<1$ and equals

$$
S_{k}=\frac{(-1)^{k-2}}{(1-a)^{k}} \text {. }
$$

To prove this fact, we start from the result of the previous proposition and substitute $r-1=x$, getting

$$
S_{k}=\sum_{x \geq k-1} \frac{1}{(k-1) !} x(x-1) \cdots(x-k+2) a^{x-(k-1)} .
$$

Now, letting $n=k-1$ and $r=x$ we obtained

$$
S_{n+1}=\frac{1}{n !} \sum_{r \geq n} r(r-1) \cdots(r-n+1) a^{r-n} .
$$

On the other hand, for $|a|<1$,

$$
\begin{aligned}
\frac{d}{d a^{n}}\left(\frac{1}{1-a}\right) & =\frac{d}{d a^{n}} \sum_{r \geq 0} a^{r}=\sum_{r \geq 0} r(r-1) \cdots(r-n+1) a^{r-n} \\
& =\sum_{r \geq n} r(r-1) \cdots(r-n+1) a^{r-n},
\end{aligned}
$$

and therefore

$$
S_{n+1}=\frac{1}{n !} \frac{d}{d a^{n}}\left(\frac{1}{1-a}\right)
$$

But, by induction,

$$
\frac{d}{d a^{n}}\left(\frac{1}{1-a}\right)=\frac{(-1)^{n-1} n !}{(1-a)^{n+1}}
$$

Thus, we have, after letting $n+1=k$,

$$
S_{k}=\frac{(-1)^{k-2}}{(1-a)^{k}}
$$

which proves our claim.

We are now almost done. Indeed, from our previous considerations, we have

1. $T_{1}\left(k=3, S_{3}=\frac{-1}{(1-a)^{3}}\right)$ :

$$
\sum_{l_{1}, l_{2}, l_{3}} N^{4} p^{2} \mu^{(3)}\left[\mu^{(1)} N\right]^{l_{1}+l_{2}+l_{3}-3}=\frac{-N^{4} p^{2} \mu^{(3)}}{\left(1-\mu^{(1)} N\right)^{3}}
$$


2. $T_{2}\left(k=2, S_{2}=\frac{1}{(1-a)^{2}}\right)$ :

$$
\sum_{l_{1}, l_{2}} N^{3} p \mu^{(2)}\left[\mu^{(1)} N\right]^{l_{1}+l_{2}-2}=\frac{N^{3} p \mu^{(2)}}{\left(1-\mu^{(1)} N\right)^{2}}
$$

3. $T_{3}, T_{4}(k=3)$ :

$$
\sum_{l_{1}, l_{2}, l_{3}} N^{4} p \mu^{(1)} \mu^{(2)}\left[\mu^{(1)} N\right]^{l_{1}+l_{2}+l_{3}-3}=\frac{-N^{4} p \mu^{(1)} \mu^{(2)}}{\left(1-\mu^{(1)} N\right)^{3}}
$$

4. $T_{5}(k=2)$ :

$$
\sum_{l_{1}, l_{2}} N^{3}\left[\mu^{(1)}\right]^{2}\left[\mu^{(1)} N\right]^{l_{1}+l_{2}-2}=\frac{N^{3}\left[\mu^{(1)}\right]^{2}}{\left(1-\mu^{(1)} N\right)^{2}}
$$

5. $T_{6}\left(k=4, S_{4}=\frac{1}{(1-a)^{4}}\right)$ :

$$
\sum_{l_{1}, l_{2}, l_{3}, l_{4}} N^{5} p^{3}\left[\mu^{(2)}\right]^{2}\left[\mu^{(1)} N\right]^{l_{1}+l_{2}+l_{3}+l_{4}-4}=\frac{N^{5} p^{3}\left[\mu^{(2)}\right]^{2}}{\left(1-\mu^{(1)} N\right)^{4}}
$$

Finally, we are ready to write the formula for $\bar{\kappa}_{3}$. It reads

$$
\begin{aligned}
\bar{\kappa}_{3} & =\frac{\bar{\Lambda}}{N^{3}} \frac{-N^{4} p^{2} \mu^{(3)}}{\left(1-\mu^{(1)} N\right)^{3}}+\frac{3 \bar{\Lambda}}{N^{3}} \frac{N^{3} p \mu^{(2)}}{\left(1-\mu^{(1)} N\right)^{2}}+\frac{3 \bar{\Lambda}}{N^{3}} \frac{-N^{4} p \mu^{(1)} \mu^{(2)}}{\left(1-\mu^{(1)} N\right)^{3}} \\
& +\frac{6 \bar{\Lambda}}{N^{3}} \frac{-N^{4} p \mu^{(1)} \mu^{(2)}}{\left(1-\mu^{(1)} N\right)^{3}}+\frac{6 \bar{\Lambda}}{N^{3}} \frac{N^{3}\left[\mu^{(1)}\right]^{2}}{\left(1-\mu^{(1)} N\right)^{2}}+\frac{3 \bar{\Lambda}}{N^{3}} \frac{N^{5} p^{3}\left[\mu^{(2)}\right]^{2}}{\left(1-\mu^{(1)} N\right)^{4}} .
\end{aligned}
$$

